\title{
Temporal Trends of Stroke Epidemiology in Southern Sweden: A Population-Based Study on Stroke Incidence and Early Case-Fatality
}

\author{
Joseph Aked ${ }^{a, b}$ Hossein Delavaran ${ }^{a, b}$ Bo Norrving ${ }^{a, b}$ Arne Lindgren ${ }^{a, b}$

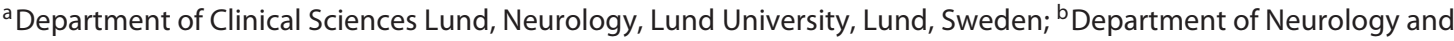 \\ Rehabilitation Medicine, Skåne University Hospital, Lund, Sweden
}

\section{Keywords}

Epidemiology $\cdot$ Stroke incidence $\cdot$ Sweden

\begin{abstract}
Background: Up-to-date epidemiological stroke studies are important for healthcare planning and evaluating prevention strategies. This population-based study investigates temporal trends in stroke incidence and case-fatality in southern Sweden. Methods: First-ever stroke cases in the local catchment area of Skåne University Hospital in Lund, Sweden, between March, 2015 and February, 2016, were included from several sources, including 2 prospective hospital-based registers, retrospective screening of primary care visits, and autopsy registers. Stroke incidence and 28-day case-fatality rates were compared with data from this area obtained through similar methodology between March, 2001 and February, 2002. Results: Altogether, 456 and 413 first-ever stroke patients were identified during the earlier and later time periods respectively. The age- and sex-standardized stroke incidence rates decreased from $246(95 \% \mathrm{Cl}$
\end{abstract}

$224-270)$ to 165 (95\% Cl 149-182) per 100,000 people. However, incidence remained unaltered among those $<65$ years. Early case-fatality decreased from 14 to $11 \%(p=0.165)$. Conclusion: First-ever stroke incidence in southern Sweden has decreased with $33 \%$ since the beginning of this millennium. Incidence rates have decreased among the elderly but remain unchanged among younger age groups. Our findings warrant further studies on trends in risk factor profiles and effects of prevention strategies, and heightened focus on stroke in the young.

(c) 2018 S. Karger AG, Basel

\section{Introduction}

Stroke constitutes a major global health problem; it is the second most common cause of death and one of the leading causes of adult disability in the world [1-3]. Moreover, stroke is accountable for more than $4 \%$ of direct healthcare costs in high-income countries [4]. Hence, as stroke has considerable human and societal repercus-

\section{KARGER}

๑c 2018 S. Karger AG, Basel

E-Mail karger@karger.com

www.karger.com/ned 
sions worldwide, it is regarded as one of the most highly prioritized non-communicable diseases by the World Health Organization (WHO) and the United Nations [5].

Recent estimates from the Global Burden of Disease (GBD) study show that the worldwide number of prevalent and incident stroke cases in 2016 amounted to nearly 83 million and 13.6 million respectively [6]. It has also been reported that the global burden of stroke is steadily increasing in absolute terms due to aging and growth of populations [3, 7]. The age-adjusted (to the World Standard Population from 2001) global incidence rate of stroke has been estimated to be 258 cases per 100,000 person-years [8]. However, there is a great deal of geographical variation in stroke incidence, and recent reports have described a decline in stroke incidence and mortality in high-income countries over the past 2 decades [9]. Furthermore, a trend toward a decline in early case-fatality of stroke has been reported in both high-income and lowand middle-income countries over the past decade, although early case-fatality rates are about $25 \%$ higher in low- and middle-income countries [9]. This implies that stroke may require regional and country-specific approaches for prevention and care [5].

A previous population-based study in southern Sweden estimated the age-adjusted (to the European Standard Population from 1976) stroke incidence rate to 144 cases per 100,000 person-years in 2001-2002 [10]. Also, early case-fatality rates of $14 \%$ have been previously reported in the south of Sweden [11]. However, these Swedish studies were performed several years ago, and there is a need for updated detailed epidemiological studies due to changing population demographics, as well as advances in stroke care and management. Up-to-date and reliable data regarding stroke epidemiology are essential for optimal healthcare planning including optimized preventive approaches [5].

We therefore used suggested gold standard criteria for epidemiological stroke studies [12] to examine the population-based stroke incidence and early case-fatality rates in southern Sweden between 2015 and 2016, as well as temporal trends since the beginning of this millennium.

\section{Subjects and Methods}

This study was approved by the Regional Ethical Review Board in Lund, Sweden (number 2016/179).

\section{Study Area and Population}

The study area comprises 8 municipalities in southern Sweden that constitute the local catchment area of Skåne University Hospital in Lund (SUS Lund), with a total population of 274,239 in- habitants as of December 31,2015 [13]. The proportion of the population $\geq 75$ years was $8 \%$, and $50 \%$ of the total population were female (December 31, 2015). The corresponding figures as of December 31,2001 , were 7 and $50 \%$, respectively, with a total population size of 234,505 inhabitants.

Besides the aforementioned public hospital with emergency facilities, the study area also has 27 primary care centers of which 9 belong to the private sector. Like the rest of Sweden, there is a robust tradition of in-hospital care for acute stroke patients in this area (predominantly at the stroke unit in the Department of Neurology at SUS Lund) [10]. For the present study, we aimed to include all first-ever stroke patients (both hospitalized and non-hospitalized) in the defined area during the study period between March 1, 2015 and February 29, 2016.

\section{Case Ascertainment}

Stroke was defined according to the WHO criteria [14]. To obtain a complete and population-based case ascertainment, multiple overlapping sources of data were used including both prospective and retrospective methods.

First-ever stroke patients from the catchment area of SUS Lund are consecutively included in the Lund Stroke Register (LSR), as part of an ongoing prospective and longitudinal observational stroke study since March 1, 2001. The LSR has been previously described in detail $[10,15]$. To detect first-ever stroke patients, research nurses in the LSR perform "hot pursuit" of stroke cases through daily weekday screenings of patient lists from the Emergency Department as well as in- and outpatient lists at the Department of Neurology at SUS Lund. The medical records of all patients included in the LSR during the study period were reviewed by one of the authors (J.A.) for case validation.

Stroke patients in Sweden are also continuously included in the Swedish National Stroke Register (Riksstroke), a national hospitalbased register, which uses prospective and retrospective methods to include both first-ever and recurrent stroke cases [16]. Medical records and research notes of all patients admitted to SUS Lund during the study period and included in Riksstroke were reviewed by one of the authors (J.A.) to determine which additional patients with first-ever stroke (besides those already included in the LSR) were eligible for the present study.

Additionally, a retrospective search was performed in a regional database administered by the regional health authority in Region Skåne of which SUS Lund is a part. This database registers all primary care visits in the study area, as well as all outpatient clinic visits at SUS Lund. Medical records of all patients with a diagnosis code of G45, G46, G81, G83, or I60-69 according to the 10th revision of the International Classification of Diseases upon a primary care visit in the area, or an outpatient clinic visit at SUS Lund, were assessed by one of the authors (J.A.) for case validation. This retrospective screening was expanded to include all visits up to 2 months after the end of the study period to minimize the risk of missing patients with a delay until their first healthcare contact after ictus. Due to regulatory restrictions, access was denied to the records of the 9 private primary care centers (out of totally 27 primary care centers) in the area.

Finally, retrospective screening was performed in the databases of the Department of Pathology at SUS Lund and the De- 
partment of Forensic Medicine by physicians at these facilities to compile records of patients with the date of death during the study period and findings of acute cerebral infarction, intracerebral hemorrhage (ICH), or subarachnoid hemorrhage (SAH) at autopsy. These patients' medical records and autopsy reports were also assessed by one of the authors (J.A.) for case validation.

\section{Stroke Classifications}

Stroke was classified into main pathological subtypes as follows: ischemic stroke (IS); ICH; SAH; and stroke not known as ischemic or hemorrhagic [17]. This classification was verified through CT and/or MR imaging of the brain or autopsy reports. In patients with $\mathrm{SAH}$ and negative neuroimaging, lumbar puncture had been performed to confirm the diagnosis.

Clinical syndromes of IS were classified according to the $\mathrm{Ox}$ fordshire Community Stroke Project by 2 of the authors (J.A. and H.D.) as follows: total anterior circulation syndrome; partial anterior circulation syndrome; posterior circulation syndrome; and lacunar syndrome [18].

The pathogenetic mechanisms of IS were classified using the Causative Classification System for IS (CCS) version 2.0 by 2 of the CCS-certified authors (J.A. and H.D.) as follows: cardio-aortic embolism; large artery atherosclerosis ; small artery occlusion, other causes; and undetermined [19].

\section{Other Assessments}

Stroke severity in the acute phase was assessed using the National Institutes of Health Stroke Scale (NIHSS) [20, 21]. For cases included in the LSR and Riksstroke, NIHSS scoring was performed retrospectively by NIHSS-certified research nurses through the review of medical records [21]. Research nurses usually met the patients in person upon inclusion, and could then use own observations to complement the information available in the medical records. In cases where NIHSS-scoring had not been performed previously, this was also done retrospectively by one of the NIHSS-certified authors (J.A.) through review of medical records [21].

\section{Case-Fatality}

The 28-day case-fatality was defined as the proportion of included patients who were deceased within 28 days of follow-up. Information on vital status of included patients, and date of death for those who were deceased, was retrieved from the official Swedish Population Register [13].

\section{Statistics}

Age- and sex-standardized incidence rates were calculated using the direct method and with 95\% CI assuming Poisson distribution. Incidence rates were standardized to the European Standard Population from 2013 [22], and to the Swedish population as of December 31, 2015 [13].

The stroke incidence rates in the present study were compared with previously published incidence rates in the same area during 2001-2002 [11]. For analysis of temporal trends, these previous incidence rates were age- and sex-standardized to the Swedish population as of December 31, 2015 [13].

Chi-square test and Mann-Whitney U test were used for comparisons of categorical and continuous variables respectively. Logrank test was used to analyze potential differences in case-fatality rates between 2001-2002 and 2015-2016. $p$ values $<0.05$ were considered statistically significant. The SPSS software package 23 was used for all statistical analyses.

\section{Results}

In total, 413 patients with first-ever stroke between March 1, 2015 and February 29, 2016, were detected in the local catchment area of SUS Lund. The detection rate and yield of the various screening methods for this study are presented in online supplementary Table 1 (for all online suppl. material, see www.karger.com/doi/10.1159/000487948).

The median age of the included patients was 76 (range 26-100 years) and 189 (46\%) were female. The number of patients hospitalized for their stroke was 389 (94\%), and $307(74 \%)$ were admitted within $24 \mathrm{~h}$ of the onset of symptoms. The most common main pathological subtype was IS $(n=334 ; 81 \%)$, followed by ICH $(n=60 ; 15 \%)$ and SAH $(n=14 ; 3 \%)$. In 5 patients $(1 \%)$, the main pathological subtype was unknown. The proportional frequency of the main pathological stroke subtypes among patients $<65$ years as well as those $\geq 65$ years is presented in online supplementary Table 2.

Among the patients with IS, partial anterior circulation syndrome was the most frequent Oxfordshire Community Stroke Project subtype $(n=153,46 \%)$. The most common pathogenetic mechanism of IS, as evaluated with CCS, was undetermined ( $n=144,43 \%)$, followed by cardio-aortic embolism ( $n=104,31 \%)$. Detailed patient characteristics are presented in Table 1.

\section{Stroke Incidence}

The total age- and sex-standardized (adjusted to the European Standard Population 2013) stroke incidence rate was 167 (95\% CI 151-184) per 100,000 person-years in 2015-2016. The corresponding rate adjusted to the Swedish population in December 31, 2015, was 165 (95\% CI 149-182) per 100,000 person-years. The stroke incidence rate increased with advancing age and was higher for men than women across all age groups except the 7584 years age range. The age- and sex-specific, as well as age- and sex-standardized, incidence rates of first-ever stroke are presented in detail in Table 2.

The total age-adjusted (to the European Standard Population 2013) incidence rate of IS was 107 (95\% CI 91126) per 100,000 person-years among women and 165 (95\% CI 142-191) per 100,000 person-years among men. Hemorrhagic stroke (ICH and SAH) had a total age-adjusted incidence rate (to the European Standard Popula- 
Table 1. Patient characteristics

\begin{tabular}{|c|c|c|c|}
\hline Variable & 2001-2002 & 2015-2016 & $p$ value \\
\hline Female gender, $n(\%)$ & $200(44)$ & $189(46)$ & $0.573^{*}$ \\
\hline Age, years, median (IQR) & $76(67-84)$ & $76(67-84)$ & $0.808^{\dagger}$ \\
\hline NIHSS at baseline, median (IQR) & $4(2-10)$ & $5(2-10)$ & $0.437^{\dagger}$ \\
\hline Source of detection, $n(\%)^{\ddagger}$ & & & $0.029^{*}$ \\
\hline Hospital-based registers & $412(90)$ & $387(94)$ & \\
\hline Other methods & $3(1)$ & - & \\
\hline Main pathological subtype, $n(\%)$ & & & $0.001^{*}$ \\
\hline IS & $364(80)$ & $334(81)$ & \\
\hline $\mathrm{ICH}$ & $46(10)$ & $60(14)$ & \\
\hline SAH & $17(4)$ & $14(3)$ & \\
\hline Unknown & $29(6)$ & $5(1)$ & \\
\hline POCS & $77(17)$ & $53(16)$ & \\
\hline CCS subtype (IS), $n(\%)$ & & & - \\
\hline $\mathrm{CE}$ & - & $104(31)$ & \\
\hline LAA & - & $56(17)$ & \\
\hline SAO & - & $24(7)$ & \\
\hline Other & - & $7(2)$ & \\
\hline UND & - & $144(43)$ & \\
\hline
\end{tabular}

* Chi-square test.

† Mann-Whitney U test.

‡ Patients were included in the abovementioned order, that is, a patient detected through hospital-based registers was not included again upon detection in primary care and outpatient clinic registers.

IS, ischemic stroke; ICH, intracerebral hemorrhage; SAH, subarachnoid hemorrhage; OCSP, Oxfordshire Community Stroke Project; LACS, lacunar syndrome; PACS, partial anterior circulation syndrome; TACS, total anterior circulation syndrome; POCS, posterior circulation syndrome; CCS, Causative Classification System for Ischemic Stroke; CE, cardio-aortic embolism; LAA, large artery atherosclerosis; SAO, small artery occlusion; other, other causes; UND, undetermined cause.

tion 2013) of 28 (95\% CI 20-38) per 100,000 person-years among women and 30 (95\% CI $21-42)$ per 100,000 person-years among men.

\section{Temporal Trends in Stroke Incidence}

The total age- and sex-standardized stroke incidence rate (adjusted to the Swedish population in December 2015) decreased with $33 \%$ from 246 (95\% CI 224-270) per 100,000 person-years in 2001-2002 to 165 (95\% CI 149182 ) per 100,000 person-years in $2015-2016$. The stroke incidence rate for men was 287 (95\% CI 253-324) per 100,000 person-years in 2001-2002 and 178 (95\% CI 155$203)$ per 100,000 person-years in $2015-2016$. The corresponding figures for women were 205 (95\% CI 177-235) per 100,000 person-years in 2001-2002 and 152 (95\% CI 131-175) per 100,000 person-years in 2015-2016.
The incidence rate of stroke significantly decreased between 2001-2002 and 2015-2016 among patients $\geq 65$ years, that is, from $1,077(95 \%$ CI $968-1,195)$ per 100,000 person-years to 666 (95\% CI 596-742) per 100,000 person years. However, no significant decrease in stroke incidence was noted among patients $<65$ years. The temporal trends in the incidence rate of first-ever stroke are illustrated in Figures 1 and 2.

The age- and sex-standardized incidence rate of IS decreased from 197 (95\% CI 177-218) per 100,000 person-years in 2001-2002 to 134 (95\% CI 120-149) per 100,000 person-years in 2015-2016. No significant decrease in incidence rates was noted for ICH or SAH. The age- and sex-standardized incidence rate of ICH was 25 (95\% CI 18-33) in 2001-2002 and 24 (95\% CI 18-31) in 2015-2016, while corresponding rates for SAH were 7 
Table 2. Age- and gender-specific incidence rates of first-ever stroke in the uptake area of Skåne University Hospital in Lund, Sweden

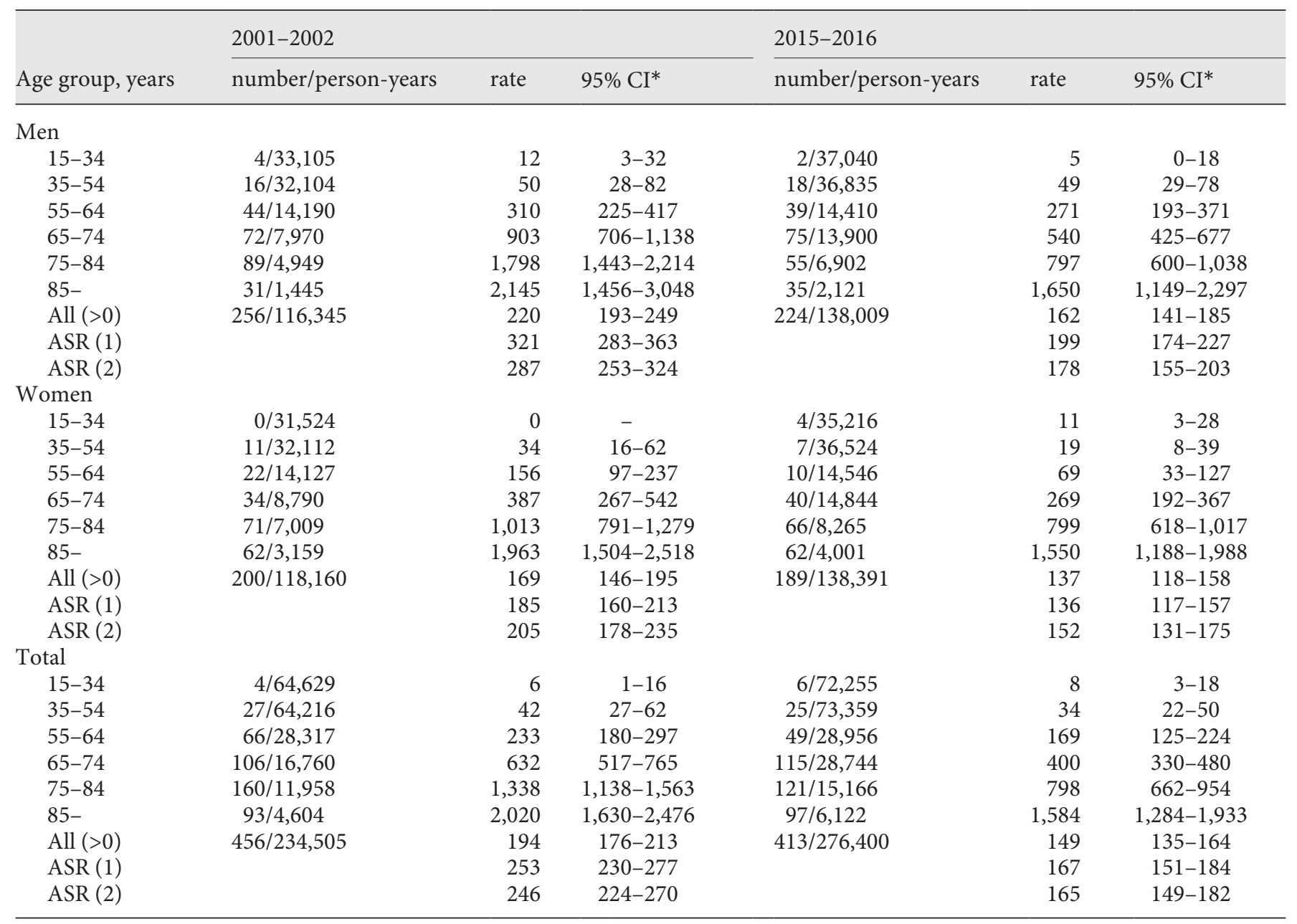

* 95\% CI were calculated assuming Poisson distribution.

ASR, age-standardized rate; (1) age-standardized to the standard European population from 2013; (2) age- and gender-standardized to the Swedish population from 2015.

(95\% CI 4-11) in 2001-2002 and 5 (95\% CI 2-8) in 2015-2016. The temporal trends in incidence rates of the main pathological subtypes of stroke are illustrated in Figure 3.

\section{Case-Fatality}

The 28-day case-fatality rate of first-ever stroke in 2015-2016 was $11 \%$. The corresponding rates for men and women were 10 and $12 \%$ respectively. The main pathological subtype of stroke with the highest 28-day case-fatality rate was ICH (23\%), followed by IS (9\%), and SAH $(0 \%)$. Patients $<75$ years had a 28 -day case-fatality rate of $8 \%$, while the corresponding rate was $14 \%$ among patients $\geq 75$ years. There were no significant differences in 28-day case-fatality rates between the periods 2001-2002 and 2015-2016 (Table 3).

\section{Discussion}

Our study provides new population-based data on stroke epidemiology in the south of Sweden. We found that the overall incidence rate of first-ever stroke in southern Sweden decreased by approximately one third since the beginning of this millennium. The absolute number of first-ever stroke cases also decreased by nearly $10 \%$, despite population growth and aging demographics. 
Fig. 1. Temporal trends in the incidence of first-ever stroke in the uptake area of Skåne University Hospital in Lund, Sweden. Incidence rates are adjusted to the Swedish population as of December 31, 2015. Error bars represent 95\% CI assuming the Poisson distribution.

Fig. 2. Temporal trends in first-ever stroke incidence rates across various age groups in the local uptake area of Skåne University Hospital in Lund, Sweden.

Fig. 3. Temporal trends in age- and sexstandardized incidence rates of main pathological stroke subtypes in the local uptake area of Skåne University Hospital in Lund, Sweden. IS, ischemic stroke; ICH, intracerebral hemorrhage; SAH, subarachnoid hemorrhage. Incidence rates are adjusted to the Swedish population as of December 31, 2015. Error bars represent 95\% CI assuming Poisson distribution.
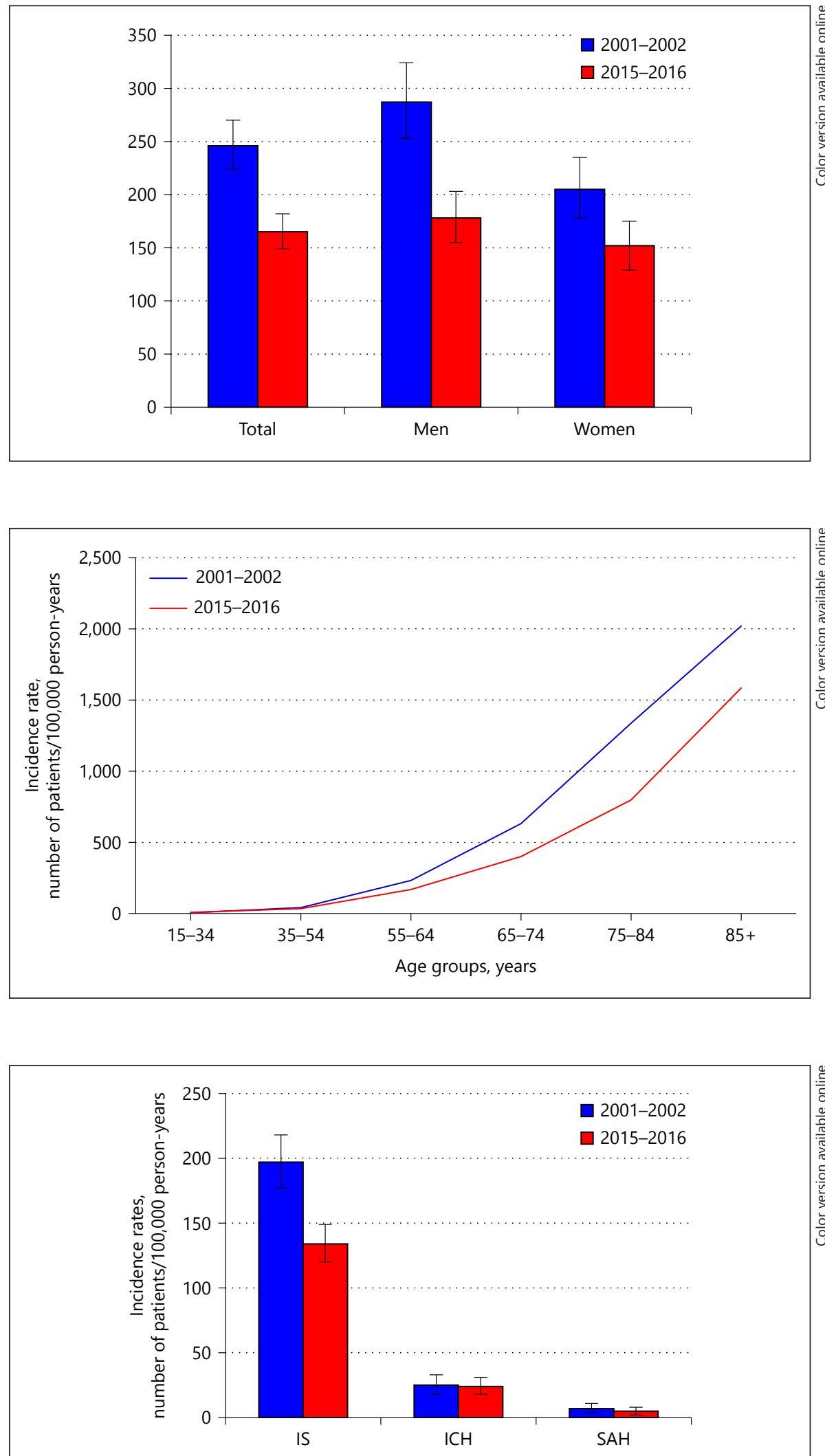
Table 3. Early case-fatality rates of first-ever stroke in the uptake area of Skåne University Hospital in Lund, Sweden

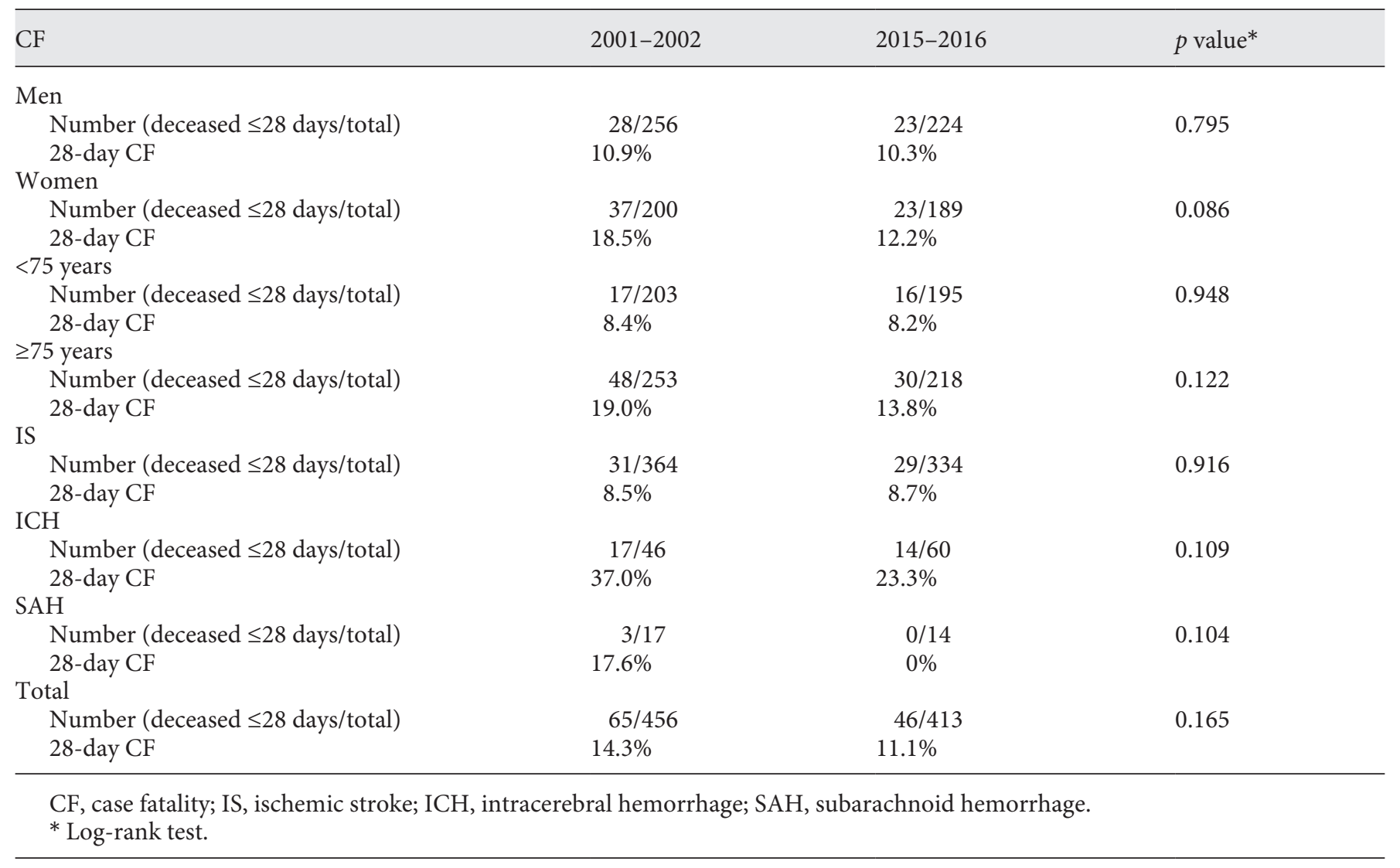

However, the stroke incidence remained unaltered among younger age groups. Moreover, early case-fatality rates did not change significantly between 2001-2002 and 2015-2016.

The 33\% decrease in stroke incidence rates between 2001-2002 and 2015-2016 is in line with findings in the comprehensive GBD study [3], and other recent epidemiological stroke studies [9]. The observed decline in stroke incidence in our study was confined to IS, whereas the incidence of hemorrhagic stroke remained unchanged. Similarly, a study from Tromsø, Norway, reported a $24 \%$ decrease in first-ever IS incidence between 1995 and 2010 [23]. The absolute number of incident first-ever stroke cases decreased by almost $10 \%$ in our study area between 2001-2002 and 2015-2016 despite an increase in population size with $18 \%$, as well as a $1 \%$ increase in the proportion of individuals $\geq 75$ years. This finding is in contrast to the GBD report [3], and previously estimated stroke incidence projections for our area [11]. The decrease in stroke incidence in our region might be related to a reduced prevalence of stroke risk factors in the population as well as improved prevention measures [24]. Likewise, the Tromsø study recently reported that changes in cardiovascular risk factors explained $57 \%$ of the decline in stroke incidence between 1995 and 2012, with reductions in systolic blood pressure and prevalence of smoking being the most important contributors [25]. The decrease in stroke incidence in our study was most prominent among patients $\geq 65$ years, which may also be related to increased use of oral anticoagulant therapy in older age groups with atrial fibrillation [26]. Other contributing factors might be improved routines for investigation and secondary prevention of transient ischemic attacks; more widespread use of acute treatments such as thrombolysis and thrombectomy (which in some cases may accomplish full regression of focal neurological symptoms within $24 \mathrm{~h}$ ) [27]; as well as heightened public awareness of cerebrovascular diseases, which may have increased the number of patients seeking urgent medical attention for conditions such as transient ischemic attacks [28]. Taken together, our findings of declining 
stroke incidence in absolute terms, despite population growth and aging demographics, warrant more research regarding current preventive approaches, to develop region-specific stroke prevention programs that are crucial to impede the otherwise globally growing burden of stroke [3].

For individuals $<65$ years, we found no significant change in stroke incidence between 2001-2002 and 20152016. Other studies have reported similar findings [29] as well as increased stroke incidence among younger people since the beginning of the 21st century [30,31]. This may be related to increased prevalence of stroke risk factors among younger people [31]. Also, recent stroke prevention interventions may have primarily been targeted at the elderly population. Additionally, different proportional frequencies of main pathological stroke subtypes in young individuals compared to the elderly may also contribute (online suppl.Table 2), since rates of IS have decreased in recent years, while corresponding rates for hemorrhagic stroke have remained unchanged [32]. Altogether, our findings of unaltered stroke incidence among the young suggest that targeted public health interventions that also include younger people is a crucial component in the efforts to tackle the overall burden of stroke.

Early case-fatality rates in our study showed a nonsignificant decline between 2001-2002 and 2015-2016. Other recent studies from high-income countries have reported similar results, wherein 28-day case-fatality after stroke has either declined or remained stable [9]. A decline in early case-fatality rates after stroke could be related to improved acute therapies and management of stroke, as well as changes in the stroke panorama with less severe strokes as a result of better preventive measures and higher detection rate of minor strokes (due to enhanced diagnostic methods) [23]. However, we found no significant difference in stroke severity between 20012002 and 2015-2016 (Table 1).

Strengths of the present study include populationbased and prospective design, hot and cold pursuit of stroke cases, inclusion of both hospitalized and nonhospitalized patients, and a well-defined study population with continuously updated population statistics. However, one limitation is that 9 private primary care centers in the study area were not included in the screening for possible stroke cases due to regulatory restrictions. Nonetheless, upon extrapolation of the current case ascertainment rate of $0.8 \%$ (95\% CI 0.4-1.4) in primary care, only 4 (95\% CI 3-8) additional firstever stroke patients are estimated to have been missed (online suppl. Table 1). It cannot be ruled out that stroke patients in the private sector of primary care may represent younger patients with milder strokes. However, in the prior study from 2001-2002 in our area, possible stroke cases within the primary care of one district (Orup, 56,565 inhabitants) were not screened due to the absence of computerized medical records at the time but were screened for the present study [10]. Also, our study is limited by a somewhat small population size, including relatively few young stroke patients. Finally, analyses of stroke risk factors have not been performed.

In conclusion, the first-ever stroke incidence has declined in southern Sweden since the beginning of this millennium, both in terms of overall incidence rates as well as absolute numbers of incident strokes, despite population growth and aging demographics. However, the stroke incidence rates have remained unaltered among younger individuals. Early case-fatality rates were also unchanged. Our findings warrant further research to delineate the underlying causes to enable optimized strokeprevention strategies, and also emphasize the need for a heightened focus on stroke in the young.

\section{Acknowledgments}

This study was supported by the Swedish Heart and Lung Foundation; Region Skåne; Skåne University Hospital; the Freemasons Lodge of Instruction EOS, Lund; Lund University; the Foundation of Färs and Frosta - one of Sparbanken Skåne's ownership Foundations; and the Swedish Stroke Association. We would also like to thank Dr. Björn Hallström for previous works on stroke epidemiology in southern Sweden. We thank the Department of Pathology at SUS Lund, the Department of Forensic Medicine, representatives for primary care facilities in our study area, and Riksstroke for their cooperation in data collection for this manuscript.

References

1 GBD 2016 Causes of Death Collaborators: Global, regional, and national age-sex specific mortality for 264 causes of death, 1980-2016: a systematic analysis for the Global Burden of Disease Study 2016. Lancet 2017;390:11511210.

2 GBD 2016 DALYs and HALE Collaborators: Global, regional, and national disability-adjusted life-years (DALYs) for 333 diseases and injuries and healthy life expectancy (HALE) for 195 countries and territories, 1990-2016: a systematic analysis for the Global Burden of Disease Study 2016. Lancet 2017;390:12601344. 
3 Feigin VL, Krishnamurthi RV, Parmar P, Norrving B, Mensah GA, Bennett DA, et al: Update on the global burden of ischemic and hemorrhagic stroke in 1990-2013: the GBD 2013 Study. Neuroepidemiology 2015;45:161-176.

4 Rothwell PM, Algra A, Amarenco P: Medical treatment in acute and long-term secondary prevention after transient ischaemic attack and ischaemic stroke. Lancet 2011;377:1681-1692.

5 Feigin VL, Norrving B, George MG, Foltz JL, Roth GA, Mensah GA: Prevention of stroke: a strategic global imperative. Nat Rev Neurol 2016;12:501-512.

6 GBD 2016 Disease and Injury Incidence and Prevalence Collaborators: Global, regional, and national incidence, prevalence, and years lived with disability for 328 diseases and injuries for 195 countries, 1990-2016: a systematic analysis for the Global Burden of Disease Study 2016. Lancet 2017;390:1211-1259.

7 Roth GA, Forouzanfar MH, Moran AE, Barber R, Nguyen G, Feigin VL, et al: Demographic and epidemiologic drivers of global cardiovascular mortality. N Engl J Med 2015; 372:1333-1341.

8 Feigin VL, Forouzanfar MH, Krishnamurthi R, Mensah GA, Connor M, Bennett DA, et al: Global and regional burden of stroke during 1990-2010: findings from the Global Burden of Disease Study 2010. Lancet 2014;383:245-254.

9 Feigin VL, Lawes CM, Bennett DA, BarkerCollo SL, Parag V: Worldwide stroke incidence and early case fatality reported in 56 population-based studies: a systematic review. Lancet Neurol 2009;8:355-369.

10 Hallström B, Jönsson AC, Nerbrand C, Petersen B, Norrving B, Lindgren A: Lund Stroke Register: hospitalization pattern and yield of different screening methods for firstever stroke. Acta Neurologica Scandinavica 2007;115:49-54.

11 Hallström B, Jönsson AC, Nerbrand C, Norrving B, Lindgren A: Stroke incidence and survival in the beginning of the 21st century in southern Sweden: comparisons with the late 20th century and projections into the future. Stroke 2008;39:10-15.
12 Sudlow CL, Warlow CP: Comparing stroke incidence worldwide: what makes studies comparable? Stroke 1996;27:550-558.

13 Statistics Sweden: Statistikdatabasen. Stockholm. http://www.scb.se (accessed May 12, 2017).

14 Hatano S: Experience from a multicentre stroke register: a preliminary report. Bull World Health Organ 1976;54:541-553.

15 Delavaran H, Jönsson AC, Lövkvist H, Iwarsson S, Elmståhl S, Norrving B, et al: Cognitive function in stroke survivors: a 10-year followup study. Acta Neurologica Scandinavica 2017;136:187-194.

16 Sveriges Kommuner och Landsting: Nationella kvalitetsregister inom hälso- och sjukvården 2007, (National quality registers in healthcare 2007; in Swedish).

17 Sudlow CL, Warlow CP: Comparable studies of the incidence of stroke and its pathological types: results from an international collaboration. International Stroke Incidence Collaboration. Stroke 1997;28:491499.

18 Bamford J, Sandercock P, Dennis M, Burn J, Warlow C: Classification and natural history of clinically identifiable subtypes of cerebral infarction. Lancet 1991;337:1521-1526.

19 Ay H, Benner T, Arsava EM, Furie KL, Singhal $A B$, Jensen $M B$, et al: $A$ computerized algorithm for etiologic classification of ischemic stroke: the Causative Classification of Stroke System. Stroke 2007;38:29792984.

20 Lyden P: Using the National Institutes of Health Stroke Scale: a cautionary tale. Stroke 2017;48:513-519.

21 Kasner SE, Chalela JA, Luciano JM, Cucchiara BL, Raps EC, McGarvey ML, et al: Reliability and validity of estimating the NIH stroke scale score from medical records. Stroke 1999; 30:1534-1537.

22 European Commission: Revision of the European Standard Population - Report of Eurostat's task force, 2013.

23 Vangen-Lönne AM, Wilsgaard T, Johnsen SH, Carlsson M, Mathiesen EB: Time trends in incidence and case fatality of ischemic stroke: the Tromsö study 1977-2010. Stroke 2015;46:1173-1179.

24 George MG, Tong X, Kuklina EV, Labarthe DR: Trends in stroke hospitalizations and associated risk factors among children and young adults, 1995-2008. Ann Neurol 2011; 70:713-721.

25 Vangen-Lönne AM, Wilsgaard T, Johnsen SH, Lochen ML, Njolstad I, Mathiesen EB: Declining incidence of ischemic stroke: what is the impact of changing risk factors? The Tromso Study 1995 to 2012. Stroke 2017;48: 544-550.

26 Mochalina N, Isma N, Svensson PJ, Sjalander A, Carlsson M, Juhlin T, et al: Ischemic stroke rates decline in patients with atrial fibrillation as anticoagulants uptake improves: a Swedish cohort study. Thromb Res 2017; 158:44-48.

27 Peisker T, Koznar B, Stetkarova I, Widimsky P: Acute stroke therapy: a review. Trends Cardiovasc Med 2017;27:59-66.

28 Nordanstig A, Asplund K, Norrving B, Wahlgren $\mathrm{N}$, Wester $\mathrm{P}$, Rosengren L: Impact of the Swedish National Stroke Campaign on stroke awareness. Acta Neurologica Scandinavica 2017;136:345-351.

29 Wang Y, Rudd AG, Wolfe CD: Age and ethnic disparities in incidence of stroke over time: the South London Stroke Register. Stroke 2013;44:3298-3304.

30 Kissela BM, Khoury JC, Alwell K, Moomaw CJ, Woo D, Adeoye O, et al: Age at stroke: temporal trends in stroke incidence in a large, biracial population. Neurology 2012;79: 1781-1787.

31 Rosengren A, Giang KW, Lappas G, Jern C, Toren K, Bjorck L: Twenty-four-year trends in the incidence of ischemic stroke in Sweden from 1987 to 2010. Stroke 2013;44:23882393.

32 Cabral NL, Cougo-Pinto PT, Magalhaes PS, Longo $\mathrm{AL}$, Moro $\mathrm{CH}$, Amaral $\mathrm{CH}$, et al: Trends of stroke incidence from 1995 to 2013 in Joinville, Brazil. Neuroepidemiology 2016; 46:273-281. 